\title{
Bacterial Biofilm: Dispersal and Inhibition Strategies
}

\author{
Shadia M. Abdel-Aziz ${ }^{1^{*}}$ and Aeron $\mathrm{A}^{2}$ \\ ${ }^{1}$ Microbial Chemistry Department, National Research Center, Egypt \\ ${ }^{2}$ Biosciences Department, DAV (PG) College, India
}

*Corresponding author: Shadia M. Abdel-Aziz, Microbial Chemistry Department, National Research Center, Egypt, E-mail: abdelaziz.sm@gmail.com

Citation: Shadia M. Abdel-Aziz, Aeron A (2014) Bacterial Biofilm: Dispersal and Inhibition Strategies. SAJ Biotechnol 1: 105. doi: 10.18875/2375-6713.1.105

Article history: Received: 05 June 2014, Accepted: 16 July 2014, Published: 18 July 2014

\begin{abstract}
Bacterial biofilms are communities of microorganisms residing within a polysaccharide matrix. Dental plaque, slimy coating in tanks, and algal mats on bodies of water are examples of biofilms. It is well accepted that biofilms play important role in bacterial persistence and antibiotic resistance in chronic infections. It is acknowledged that the majority of microbial cells on earth are living in distinct communities as biofilms. In fact, it is now known that $99 \%$ of all bacteria exist in biofilms, with only $1 \%$ living in the planktonic state. It has been estimated that $65 \%$ of microbial infections are associated with biofilms which constitute a microbial multicellular lifestyle and are defined as organized communities of bacteria. The structural nature of biofilms can protect the cells against antimicrobial agents and the host's defense. The microbial community inside a biofilm can also be protected against adverse conditions such as desiccation, osmotic shock, UV radiation, or exposure to toxic compounds, or predators. Due to the heterogeneous nature of biofilms, it is likely that multiple mechanisms of antimicrobial resistance can occur.
\end{abstract}

Keywords: Biofilms; Antimicrobial agents; Pathogens; Polysaccharides; Antibiotics

\section{Introduction}

Biofilms are groups of microorganisms in which cells stick to each other on a surface. A biofilm, sometimes referred to as slime, is a polymeric mixture generally composed of extracellular DNA, proteins, and polysaccharides [1]. Bacterial polysaccharides are a major component of the extracellular polymeric substance or matrix of biofilms, and mediate most of the cell-to-cell and cellto-surface interactions required for biofilm formation and stabilization [2]. Biofilms may form on living or non-living surfaces, on solid or liquid surfaces as well as on soft tissue in living organisms, and are typically resistant to conventional methods of disinfection. Dental plaque, slimy coating in tanks, and algal mats on bodies of water are examples of biofilms. Biofilm may be detrimental or beneficial. While biofilms are generally pathogenic in the body, causing more diseases, they can be used beneficially in treating sewage, industrial waste, and contaminated soil. Biofilm formation protects and enables single-cell organisms to assume a multicellular lifestyle, in which "group behavior" facilitates survival in adverse environments [3]. Transition from planktonic growth to biofilm occurs in response to environmental changes, and involves multiple regulatory networks, which translate signals to a concerted gene expression changes. Four major antibiofilm strategies are studied to prevent detrimental biofilm formation. These include: 1) Prevention by antibiotic prophylaxis and targeting of surface molecules. 2) Weakening by degradation of extracellular matrix, inhibition of efflux pumps, or targeting of extracellular and intracellular signaling molecules. 3) Disruption by mechanical ways, biological disruption with enzymes, or targeting of extracellular and intracellular signaling molecules. 4) Killing by targeting of the bacterial membrane or targeting subpopulations with different classes of antibiotics [4]. This review deals with biofilm formation, highlighting several medically important pathogens, and discusses recent advances on novel strategies for biofilm dispersal and inhibition.

\section{A Brief History and Development}

Antonie van Leeuwenhoek (1684) was the first to display the animalcule (bacteria) found in the plaque of teeth, and described in a report to the Royal Society of London. In 1940, H. Heukelekian and A. Heller wrote about the development of bacterial slime and colonial growth attached to surfaces. Zobell (1943) reported about seawater and described many of the fundamental characteristics of attached microbial communities. Since such communities were described and named biofilms in 1978, biofilm science and 
biofilm engineering become active fields of study [5]. Microorganisms constitute the most successful form of life on earth and affect human existence and well-being either directly by influencing human health and diseases, or indirectly, by carrying out processes in the natural or man-made environments [5].

Bacteria form an architecturally complex community (Figure 1) to control cell fate [6]. Biofilms harbor multiple cell types, and it has been proposed that within biofilms individual cells follow different developmental pathways, resulting in heterogeneous populations [6]. Importantly, mutants do not produce extracellular matrix, i.e., mutants are typically deficient in adherence and biofilm formation (Figure 2). Understanding the processes that control biofilm formation is important for development of methods needed to control chronic infections [7]. Cell differentiation is ubiquitous and facilitates division and development. Bacteria are capable of multicellular behaviors that benefit the bacterial community as a whole. A striking example of cell differentiation in bacteria is the formation of a biofilm [6,8]. Micro-colonies of bacterial cells encased in polysaccharide matrix are separated from each other by water channels [9]. Liquid flow occurs in water channels, allowing diffusion of nutrients, oxygen, and even antimicrobial agents. Bacteria exist in two principal forms as free cells (planktonic state) or in biofilms. Biofilm allows cells to form long-term relationships, interact with each other and establish metabolic cooperation. The association of bacteria with a surface and the development of a biofilm can be viewed as a survival mechanism, with bacteria benefiting by acquiring nutrients and protection from biocides [5].
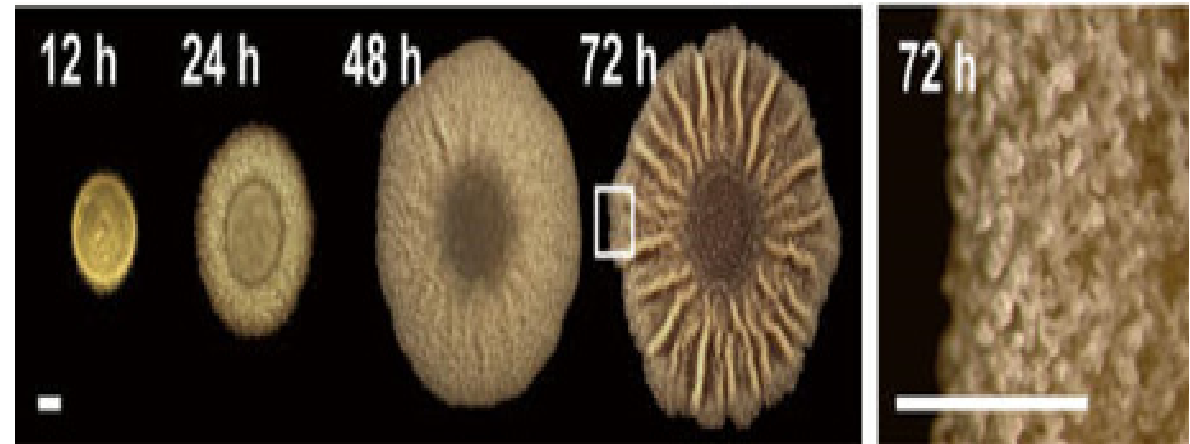

Figure 1: Top view of biofilm development over time: The right panel is magnified to highlight the structures observed at $72 \mathrm{~h}$. The population dynamics of a $B$. subtilis biofilm as a function of time were determined by harvesting samples from biofilms expressing reporters at $12,24,48$, and $72 \mathrm{~h}$ of development [6].
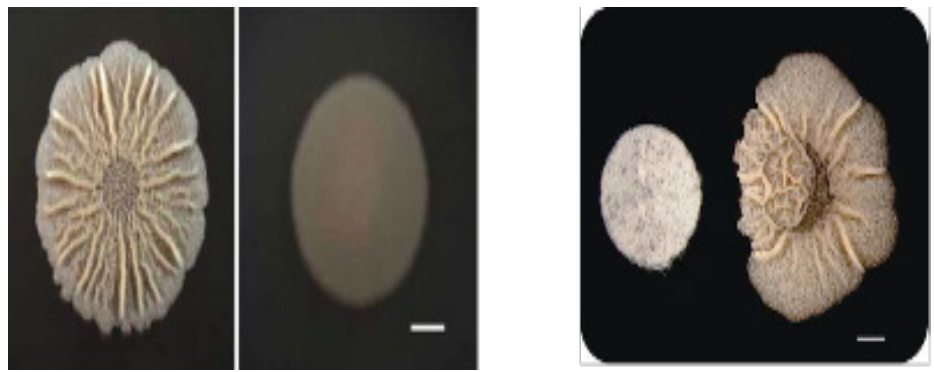

Figure 2: Colony morphology of B. subtilis strain 3610 wild type (left image) and its matrix mutant (Cells after 3-d of growth on $1.5 \%$ agar MSgg medium); and effect of the antimicrobial nisin $(0.6 \mu \mathrm{M})$ on B. subtilis biofilm morphology (right image). Cells closer to the disk containing nisin are more wrinkled due to the presence of more matrix-producing cells [10].

\section{Stages of Biofilm Development}

Biofilms consist of microorganisms and their self-produced extracellular polymeric substances (Exopolysaccharide). A fully developed biofilm contains many layers including a matrix of exopolysaccharide with vertical structures, and a conditioning film. Vertical structures of microorganisms sometimes take the form of towers or mushrooms, and are separated by interstitial spaces.

Formation of biofilms is rather complex, but can be generalized in four basic steps: 1) deposition of the conditioning film which alter the surface properties of the substratum and allow microorganisms to adhere to the surface. 2) Microbial (planktonic) attachment to the conditioning film. 3) Growth and bacterial colonization, where production of polysaccharides that anchor the bacteria to the surface allow colonies to grow [11,12] and 4) biofilm formation, where a fully developed biofilm will contain an EPS matrix and vertical structures separated by interstitial spaces (Figure 3).

Some of the cells are adsorbed to the surface for only a finite time, before being deadsorbed, in a process called "reversible adsorption" [13]. This initial attachment is based on electrostatic attraction and physical forces, but not due to any chemical attachments. Some of these reversibly adsorbed cells begin to make preparations for a lengthy stay by forming structures which may then permanently bind then to the surface within the next few hours, the pioneer cells proceed to reproduce and the daughter cells, form microcolonies on the surface and begin to produce a polymer matrix around the microcolonies, in an irreversible steps [13]. 


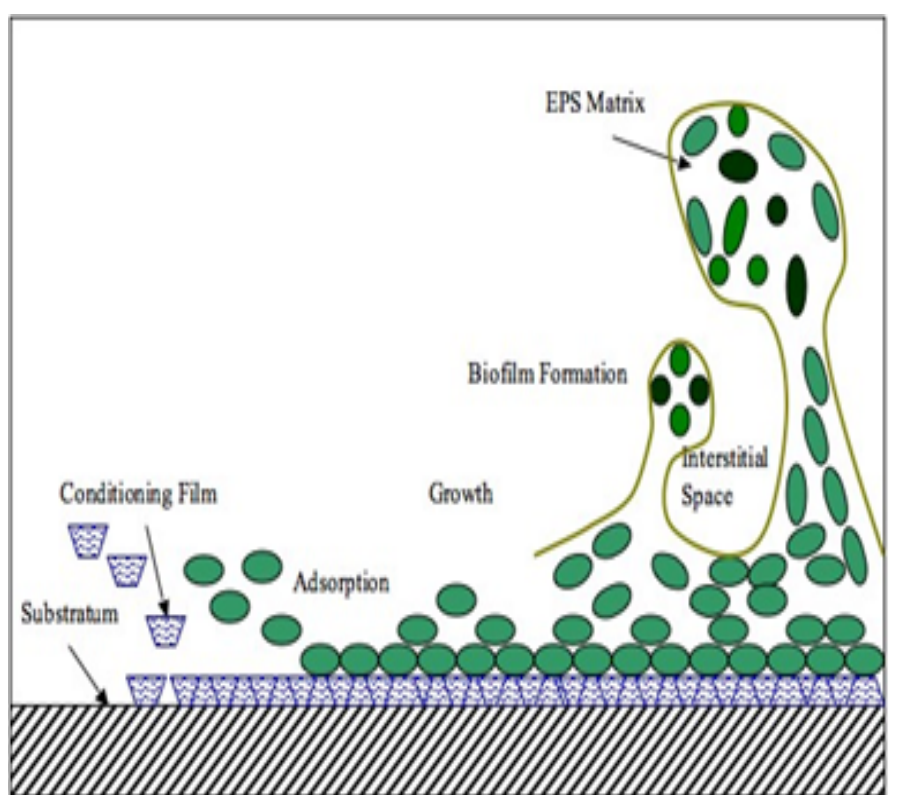

Figure 3: Stage process for development of a biofilm (Lennox 2011)

Biofilms are permeated at all levels by a network of channels through which water, bacterial garbage, nutrients, enzymes, metabolites and oxygen move to and from, with gradients of chemicals and ions between micro-zones providing the power to shunt the substances around the biofilms [5]. In a mature biofilm, more volume is occupied by the loosely organized glycocalyx matrix (7595\%) than by bacterial cells $(5-25 \%)[9,11,12]$. In most cases, the base of the biofilm is a bed of dense, with thickness up 5 to $50 \mu \mathrm{m}$, composed of a sticky mix of polysaccharides, other polymeric substances and water, all produced by the bacteria [14]. Soaring 100 to $200 \mu \mathrm{m}$ upwards are colonies of bacteria, shaped like mushrooms or cones. The development of a mature biofilm may take from several hours to several weeks, depending on the system [15].

\section{Protection of Biofilm against Antibiotics}

\section{Bacterial Biofilm}

Traditional antibiotic resistance of free-living bacteria usually involves inactivation of the antibiotic, modification of targets, and exclusion of the antibiotic [16]. These actions typically require the acquisition of specific genetic factors, such as genes for $\beta$-lactamase. One of the most important aspects of bacterial biofilm formation is the increased resistance of the constituent microbes to antibiotics and other stressors. The structural nature of the biofilm and the characteristics of the sessile cells produce resistance towards the antimicrobial agents, leading to a protected environment against adverse conditions and the host's defenses [16,17]. Free living bacteria, on other hand, are generally susceptible to antibiotic treatment and to host defense mechanisms. However, the minimal inhibitory concentration and minimal bactericidal concentration of antibiotics to biofilm-growing bacteria being up to 100 -1000 fold higher than for free bacteria, and possibly 150-3000 times more resistant to disinfectant [16]. Fundamental factors of resistance are activated as part of the biofilm developmental pathway. Influences of several different biofilm factors affecting antibiotic resistance have been identified. For example: 1) biofilm matrix may act as a diffusion barrier. 2) micro environments within biofilms can be established. 3) small subpopulations of bacteria within the biofilms may differentiate into persisters. 4) increased production of oxidative stress causes might changes in the physiology of bacteria and 5) antagonist of antibiotics and degradation mechanisms may be active in some parts of the biofilms [5].

Biofilms can act as physical diffusion barriers to prevent antibiotics from reaching their targets. Antibiotics are shown to be able to penetrate through a thick mixture of polysaccharide, DNA, and protein to reach its target [18]. Depletion of nutrients and oxygen inside biofilms might cause an altered metabolic activity and lead to slow growth of the bacteria. In general, all antimicrobials are more effective in killing rapidly growing cells. Therefore, slow growth undoubtedly contributes to biofilm resistance [5]. In addition, slow growth is a major factor in the increased resistance of stationary planktonic cells to be killed.

\section{Persister cells}

Resistance to antibiotics in both stationary phase cells and biofilms may be due to the presence of persister cells which are mechanistically distinct from multidrug resistance [19]. It is not caused by mutant microbes, but rather by microbial cells that exist in a transient, dormant, non-dividing state. Microorganisms that display multidrug tolerance can be bacteria, fungi or parasites. Multidrug tolerance or antibiotic tolerance is the ability of a disease-causing microorganism to resist killing by antibiotics or other antimicrobials [19]. Persister cells are considered to be either non-growing or slow-growing, and also have a greatly reduced susceptibility to antibiotics [19]. In the persister's theory, these small subpopulations of bacteria within the biofilms, differentiated into 
dormant cells, are able to survive extreme antibiotic treatment and have been hypothesized to be the result of phenotypic variations rather than due to stable genetic changes [20]. Indeed, the formation of persisters might represent a common mechanism used by a wide range of bacteria during biofilm formation. This persistent population within biofilms may, drastically, inhibit the complete eradication of the biofilms, even after prolonged, high-level antibiotic treatment [5].

Biofilm infections are, in a sense, very similar to planktonic infections in the absence of an immune response. The biofilm exopolymer physically protects the cells from the components of the immune system. One can explain a biofilm survival dynamic, in vivo, in which an initial application of a cidal antibiotic eradicates most of the population, leaving a small fraction of surviving persisters [19]. If the concentration of the antibiotic temporarily drops or if symptoms disappear due to the eradication of planktonic cells and therapy is discontinued, the persisters will reform the biofilm, which will begin to shed off new planktonic cells [21]. This dynamic explains the deteriorating nature of biofilm infections and the need for a lengthy antibiotic therapy. Indeed, if a biofilm of a particular species comprises fewer persister cells than a planktonic population, it will survive better than planktonic cells and will become more resistant to antibiotics and the immune attack [21]. Thus, a model of biofilm resistance based on persister survival, that is, an initial treatment with antibiotic kills planktonic cells and the majority of biofilm cells. The immune system kills planktonic persisters, but the biofilm persister cells are protected from host defenses by the exopolysaccharide matrix. After the antibiotic concentration drops, persister cells revive the biofilm and the infectious agents become more effectiveness and reinforced [21].

\section{Riddle of Biofilm Resistance}

Bacterial biofilm provides a protective mechanism for the cells to be less susceptible to antibiotics [19]. Therefore, dispersal of biofilm for removal of cells outside the matrix enables antibiotics for treatment of bacterial infections [22]. Removal of these cells from the matrix of the biofilm renders them to be susceptible to antibiotics [22]. There have been a number of models used to determine resistance in biofilms, and the results of these studies have highlighted a number of the factors thought to contribute to the ability of a biofilm to tolerate high concentrations of antibiotics [22]. These suggested mechanisms include:

1) Impaired penetration of an antibiotic into the biofilm matrix may be an explanation for biofilm resistance to antibiotic [23].

2) Antibiotics are more effective in killing cells when they are growing actively. Therefore, inside the biofilm, bacteria physiologically change from being active in the planktonic state to the immobile and non-growing state inside the biofilm [14].

3) Altered micro-environment within the biofilm (e.g., pH, oxygen content), which reduces the activity of an antimicrobial agent. There is evidence of gradients of physiological activity within a biofilm in response to antibiotic treatment [24]. This would suggest that the response to antibiotics will vary according to the location of specific cells within a biofilm ecosystem [22].

4) Altered gene expression: altered gene expression by organisms within a biofilm or a general stress response of a biofilm has been documented as factors known to reduce susceptibility to antibiotics [25].

5) Reduced biofilm-specific phenotype: it has been suggested that a biofilm-specific phenotype may be induced in a subpopulation of the biofilm [26]. These subpopulations have been shown to express active mechanisms to reduce the efficacy of antibiotics [26].

\section{Biofilms and infectious diseases}

Native bacterial communities populate human mucous membranes and epithelial surfaces such as the gastrointestinal tract, oral cavity, and skin. These bacterial communities play key role in the immune systems and protection of the mucosal surfaces, and also provide vital functions against exogenous pathogens [27]. The relationship between the host and its microbial communities is carefully balanced, but under certain conditions it can break down and result in infectious diseases. According to a recent public announcement from the National Institutes of Health, more than 60\% of all microbial infections are caused by biofilms. Some human diseases involving biofilms [28] are summarized in Table 1. Presence of biofilm confirms an important role for bacterial chronic infection associated with antibiotic resistance.

Growth of biofilm in food processing environments leads to opportunity for microbial contamination of the processed food. Microorganisms within the biofilm are protected from sanitizers, therefore: their survival and chance to contaminate foods are also increased. The most prevalent strain, Listeria monocytogenes, among pathogenic bacteria (Table 2) that found in the food processing environments has good adhesion ability and requires only a short contact time for attachment [29]. Biofilm control efforts most often focus on effective cleaning of potential growth sites.

Biofilm on indwelling medical devices may be composed of Gram-positive or Gram-negative bacteria or other microorganisms which may originate from the skin of patients, health-care workers, tap water or other sources in the environments. Microorganisms commonly associated with biofilm on indwelling devices [29] are shown in Table 3. 


\begin{tabular}{|c|c|}
\hline Human Disease & Biofilm-forming Bacteria \\
\hline Cystic fibrosis pneumonia & P. aeruginosa and Burkholderia cepacia \\
\hline Meloidosis & Pseudomonas pseudomallei \\
\hline Necrotizing fasciitis & Group A streptococci \\
\hline Musculoskeletal infections & Staphylococci and other Gram-positive cocci \\
\hline Otitis media & Haemophilus influenzae (Non-typable strains) \\
\hline Biliary tract infection & E. coli and other enteric bacteria \\
\hline Urinary catheter cystitis & E. coli and other Gram-negative rods \\
\hline Bacterial prostatitis & E. coli and other Gram-negative bacteria \\
\hline Periodontitis & Gram negative anaerobic oral bacteria \\
\hline Dental caries & Streptococcus spp. and other acidogenic Gram positive cocci \\
\hline
\end{tabular}

Table 1: Some human disease associated with bacteria biofilms

\begin{tabular}{|c|c|}
\hline Food-borne pathogens & Growing surface \\
\hline Listeria monocytogenes & Dairy processing plant, conveyor belt \\
\hline Pseudomonas spp. & Drain, vegetable and meat surface \\
\hline Bacillus spp. & Pepelie, joint in processing environment, hot fluid \\
\hline Salmonella spp. & Poultry processing environment \\
\hline
\end{tabular}

Table 2: Food-borne pathogens and spoilage bacteria in biofilm

\begin{tabular}{|c|c|}
\hline Medical Devices & Causative organism \\
\hline $\begin{array}{l}\text { "Urinary catheter, Intra-urine device, Prosthetic heart } \\
\text { valve, Central venous catheter }\end{array}$ & Coagulase-negative Staphylococci \\
\hline "Urinary catheter, Central venous catheter & Klebsiella pneumoniae \\
\hline $\begin{array}{l}\text { Artificial hip prosthesis, Central venous catheter, Intra- } \\
\text { urine device }\end{array}$ & Pseudomonas aeruginosa \\
\hline $\begin{array}{l}\text { "Artificial voice prosthesis, Central venous catheter, } \\
\text { Intra-urine device }\end{array}$ & Candida albicans \\
\hline $\begin{array}{l}\text { Artificial hip prosthesis, Central venous catheter, Intra- } \\
\text { urine device, Prosthetic heart valve }\end{array}$ & Staphylococcus aureus \\
\hline $\begin{array}{l}\text { "Artificial hip prosthesis, Prosthetic heart valve, Urinary } \\
\text { catheter }\end{array}$ & Enterococcus spp. \\
\hline
\end{tabular}

Table 3: Microorganisms associated with biofilm on indwelling medical devices

Biofilm-growing bacteria cause chronic infections, including foreign-body infections, that are characterized by persistent inflammation and tissue damage despite antibiotic therapy and the innate and adaptive immune and inflammatory responses of the host and persisting pathology [30]. Some general features of biofilm infections in human include: 1) Aggregates of bacteria embedded in a self-produced polymer matrix. 2) Tolerant to both innate and adaptive immune responses. 3) Tolerant to clinically dosing of antibiotics despite susceptibility of planktonic cells and 4) Chronic infections. The surface-associated microorganisms are responsible for a several chronic infections as: periodontitis [31], heart valves (endocarditis) [32], in lung infection in patients with cystic fibrosis causing chronic bronchopneumonia by Pseudomonas aeruginosa [33], child middle-ear infections (caused by Haemophilus influenzae, for example), in chronic osteomyelitis and infections caused by a variety of surgical implants [16], wound infection in burn patients, urinary tract infections (caused by Escherichia coli and other pathogens) [34], in intravenous catheters and stents (caused by Staphylococcus aureus and other gram-positive pathogens) [35]. To date, the link between the concept of biofilms and chronic infectious disease is still the subject of many studies $[8,36]$. Some pathogenic bacteria form biofilms are discussed below.

\section{Streptococcus mutans}

Dental plaque is an oral biofilm that adheres to the teeth and consists of many species of both fungal and bacterial cells (such as Streptococcus mutans and Candida albicans), salivary polymers and microbial extracellular products. The accumulation of microorganisms subjects the teeth and gingival tissues to high concentrations of bacterial metabolites which results in dental disease [37]. The biofilm on the surface of teeth is frequently subjected to oxidative stress and acid stress. Dietary carbohydrates can cause a dramatic decrease in $\mathrm{pH}$ in oral biofilms to values of 4 and below (acid stress). A pH of 4 at body temperature of $37^{\circ} \mathrm{C}$ causes depurination of DNA, leaving apurinic sites in DNA, especially loss of guanine [38]. When the oral strain $S$. mutans, inside a biofilm, exposed to an acid stress, it competes with induction of regulator factors (regulons) that aid the strain to be more resistant 
for such acid stress [9].

On other side, periodontal diseases are infections involve supporting tissue of teeth, gums (gingiva), and periodontal tissues (gingiva, alveolar bone, and periodontal ligament). Chronic periodontitis may lead to exfoliation of the teeth [29]. The main microbes associated with periodontitis are Pseudomonas anerobicus, Porphyromonas gingivalis, and Eubacterium timidum. These bacteria can colonize a number of surfaces in the oral cavity, including various mucosal surfaces [39] and the tooth surfaces. Colonization of surfaces may permit the bacteria to invade mucosal cells, alter calcium flux in epithelial cells, and release toxins. Human saliva contains a number of physical, physicochemical, and chemical agents that protect oral tissues against oral microorganisms. When dental-plaque mass increased, the antimicrobial properties of saliva become less able to penetrate and protect the tooth enamel which lead to occurrence of dental carries or periodontal diseases [29].

\section{Streptococcus pneumoniae}

Streptococcus pneumoniae is the main cause of community-acquired pneumonia and meningitis in children and the elderly. When $S$. pneumonia grows in biofilms, genes are specifically expressed that respond to oxidative stress and induce competence [40]. Formation of a biofilm depends on competence stimulating peptide which also functions as a quorum-sensing peptide. Beside induces biofilm formation, it also increases virulence in pneumonia and meningitis. It has been proposed that competence development and biofilm formation is an adaptation of $S$. pneumoniae to survive the defenses of the host [41]. In particular, the host's polymorphonuclear leukocytes produce an oxidative burst to defend against the invading bacteria, and this response can kill bacteria by damaging their DNA. Competent $S$. pneumoniae in a biofilm have the survival advantage that they can more easily take up transforming DNA from nearby cells in the biofilm to use for recombinational repair of oxidative damages in their DNA. Competent S. pneumoniae can also secrete an enzyme (murein hydrolase) that destroys non-competent cells (fratricide) causing DNA to be released into the surrounding medium for potential use by the competent cells [42].

\section{Pseudomonas aeruginosa}

Biofilms are not always less susceptible to antibiotics. For instance, the biofilm formed by Pseudomonas aeruginosa has no greater resistance to antimicrobials than do stationary-phase planktonic cells, although when the biofilm is compared to logarithmic phase planktonic cells, the biofilm does have greater resistance to antimicrobials. However, the opportunistic gram-negative pathogen, $P$. aeruginosa causes both chronic and acute infections and is one of the leading causes of morbidity and mortality in thermally injured patients [43]. The differentiation or maturation of $P$. aeruginosa biofilms in vitro depends on intercellular signaling systems or quorum sensing (QS). QS systems in many gram-negative bacteria rely on acylated homoserine lactones which are produced at high levels when cell density is high and act as ligands for transcriptional regulators. These transcriptional regulators then regulate the transcription of many genes whose products, including proteases, elastases, toxins, and hemolysins, are thought to be crucial for virulence [43]. P. aeruginosa strains lacking functional QS systems are less virulent than wild-type strains [44] and form flat, undifferentiated biofilms on glass surfaces [45]. These undifferentiated biofilms are less stable than the differentiated biofilms formed by wild-type P. aeruginosa as they can be easily disrupted by the detergent sodium dodecyl sulfate [45].

\section{Legionella pneumophila}

The genus Legionella is a pathogenic group of Gram negative bacteria that includes the species L. pneumophila, causing Legionellosis [46]. Legionella is common in many environments, including soil and aquatic systems, with at least 50 species and 70 serogroups identified. These bacteria are known to grow under certain conditions in biofilms, in which they are protected against disinfectants [47].

\section{Strategies for Biofilm Inhibition}

Removal of cells from the biofilm colony is an essential stage of the biofilm life cycle because it enables biofilms to spread and colonize new surfaces. Strategies to plan against bacterial biofilm must be achieved by prevention of biofilm formation rather than dispersal of the formed biofilm. Strategies for prevention of biofilm formation include both "Chemical" and "Mechanical" methods.

\section{Chemical methods}

Antimicrobial coatings: Chemical modifications are the main strategy for biofilm prevention. Antibiotics, biocides, and ion coatings are commonly used chemical methods of biofilm prevention. These methods prevent biofilm formation by interfering with the attachment and expansion of immature biofilms [48]. Typically, these coatings are effective only for a short time period (about 1 week), after which leaching of the antimicrobial agent reduces the effectiveness of the coating [48]. Use of silver coatings for antimicrobial purposes has been known. The antimicrobial property of silver is known as an oligodynamic effect, a process in which metal ions interfere with the growth and function of bacteria [49]. Several in vitro studies have confirmed the effectiveness of silver at preventing infection, both in coating form and as nanoparticles dispersed in a polymer matrix. However, application of silver in the in vivo system is associated with warnings due to the toxic effect of silver on human tissue.

Polymer modifications: Antimicrobial agents can be immobilized on device surfaces using long, flexible polymeric chains. These chains are anchored to the device surface by covalent bonds, producing non-leaching, contact-killing surfaces. One in vitro study 
found that when $\mathrm{N}$-alkylpyridinium bromide, an antimicrobial agent, was attached to a poly(4-vinyl-N-hexylpyridine), the polymer was capable of inactivating $\geq 99 \%$ of S. epidermidis, E. coli, and P. aeruginosa bacteria [50]. Dispersion forces between the polymer chains and the bacterial cells prevent bacteria from binding to the surface and initiating biofilm growth. The concept is similar to that of steric stabilization of colloids. Polymer chains are grafting to a surface via covalent bonding or adsorption.

\section{Mechanical methods}

Hydrophobicity, Surface roughness, Surface charge: Formation of a biofilm begins with the attachment of free-floating cells to a surface. These first colonists adhere to the surface initially through weak, reversible adhesion. If the colonists are not immediately separated from the surface, they can anchor themselves more permanently using cell adhesion structures such as pili [8]. Hydrophobicity also plays an important role in determining the ability of bacteria to form biofilms. Some species are not able to attach to a surface and are sometimes able to establish themselves directly to earlier colonists [50]. On other hand, some bacteria are unable to successfully form biofilms due to their limited motility. Nonmotile bacteria cannot recognize the surface or aggregate together as easily as motile bacteria.

Modification of the surface charge of polymers has also proven to be an effective means of biofilm prevention. Based on the principles of electrostatics charged particles will repel other particles of like charge. The hydrophobicity and the charge of polymeric chains can be controlled by using several backbone compounds and antimicrobial agents. Positively-charged polycationic chains enable the molecule to stretch out and generate bactericidal activity [50].

Surface roughness can also affect biofilm adhesion. Rough, high-energy surfaces are more conducive to biofilm formation and maturation, while smooth surfaces are less susceptible to biofilm adhesion. The roughness of a surface can affect the hydrophobicity or hydrophilicity of the contacting substance, which in turn affects its ability to adhere [51]. It is, thus, desirable to maintain a smooth surface on any products that may come in contact with bacteria [52].

\section{Strategies for Biofilm Dispersal}

Strategies for more effective biofilm dissolution treatments become fundamental. Mechanisms to understand the role of biofilms in chronic infections and antimicrobial resistance are important when design for new drug treatments [3]. Conventional antibiotics work by either preventing bacterial cell division (bacteriostatic) or killing the cell (bactericidal). Although over the years antibiotics have proven critical in eliminating bacterial pathogens, evidences indicate that they extensively damage the host microbiota, creating an environment where opportunistic pathogens can prevail [3]. Most recent advances in strategies are designed to prevent biofilm formation by killing the bacteria or targeting different biofilm developmental stages [3]. Some strategies and mechanisms for biofilm inhibition are discussed below.

\section{Bacterial Antibiofilm Polysaccharides}

Polysaccharides, as sugar polymers, have the capacity to act as lectin inhibitors. Lectins are proteins that specifically recognize and bind sugars without modifying these molecules. In bacteria, the primary function of lectins is to facilitate attachment or adherence of bacteria to host cells. These proteins play an important role in biofilm formation, and are essential for bacterial colonization and infection. Lectins are mainly located on the surface of bacteria cells where they can access and bind to the glycan substrates present on the surface of host cell. By competing for the sugar binding domain of lectins, polysaccharides can inhibit lectin-dependent adhesion of pathogens and biofilm formation. In fact, several plant, microbial and milk polysaccharides have been shown to block various lectins from human pathogenic bacteria by competitive inhibition [53]. Polysaccharides mediate cell-to-surface and cell-to-cell interactions that are critical for biofilm formation and stabilization. Recent evidence indicates that some bacterial exopolysaccharides inhibit or destabilize biofilm formation by other species [53]. Antibiofilm properties of polysaccharides are believed to lie on their ability to: a) alter the physical characteristics of bacterial cells or abiotic surfaces. b) act as signaling molecules that impact the gene expression patterns of susceptible bacteria. or c) competitively inhibit multivalent carbohydrate-protein interactions, thereby interfering with adhesion.

Many studies are reported about the ability of some bacterial polysaccharides to inhibit biofilm formation by several bacteria, including E. coli strains, P. aeruginosa, Klebsiella pneumoniae, Staphylococcus and Enterococcus (54). Most of these antibiofilm agents are able to inhibit the biofilm formation of a broad range of bacteria, suggesting that they may play an essential role in microbial competition and niche exclusion. Mutants unable to synthesize or export such polysaccharides are typically deficient in adherence and biofilm formation (Figure 2) and thus are highly sensitive to killing by antibiotics and host immune defenses [3].

\section{Anti-biofilm enzymes}

Enzymes that degrade biofilm extracellular matrix may play a role in biofilm dispersal and may be useful as anti-biofilm agents. $\mathrm{N}$-acetyl-D-glucosamine-1-phosphate acetyl transferase is an essential peptidoglycan and lipopolysaccharide precursor in Grampositive and Gram-negative pathogens, respectively, is among the enzymes targeted for matrix disruption [3]. Treatment with such enzymes prevented Staphylococcus and Enterococcus biofilm formation and disperse preformed biofilms in vitro [55]. For example, Dispersin-B is a glycoside hydrolase that cleaves $\beta$ 1-6 N-acetylglucosamine polymers in the bacterial peptidoglycan layer. Dispersin-B treatment has been shown to be effective against S. aureus and S. epidermidis biofilms and bacteria [56]. 


\section{Chelating Agents}

Metal cations, such as calcium, magnesium, and iron have been implicated in maintaining matrix integrity. Consistent with this observation, chelating agents have been shown to destabilize biofilm architecture besides interfering with bacterial membrane stability. For example, sodium citrate inhibited biofilm formation by several Staphylococci species in vitro [57]. In addition, tetrasodium-EDTA eradicated biofilms in an in vitro biofilm model and on explanted hemodialysis catheters, whereas disodiumEDTA, in combination with tigecyclin or gentamicin, reduced biofilm formation by Staphylococcus species and P. aeruginosa.

\section{Antimicrobial Peptides}

Antimicrobial peptides are produced by the innate immune response system and have been proposed as attractive candidates for the development of novel types of antibiotics. However, their activity spectrum and mechanism of action need to be more precisely defined before they can be considered as possible therapeutic strategies [58]. A recent work, focused on reduced biofilm formation by multidrug-resistant $P$. aeruginosa strains isolated from patients with cystic fibrosis, revealed that the bacterium was killed within preformed biofilms. Lytic peptides are another group of antimicrobial peptides assessed for their inhibitory effects on biofilm formation. Lytic peptides bind the lipopolysaccharide moieties of the bacterial cell membrane, disrupting membrane stability [58]. Studies in Staphylococcus aureus have shown that a lytic peptide prevented in vitro biofilm formation and was also capable of diffusing into the deep layer of preformed biofilm, killing $99.9 \%$ of biofilm bacteria. This peptide retained activity under highly acidic environments and in the presence of excess of metals, conditions that mimic the $S$. aureus biofilm environment.

\section{Anti-adhesion Agents}

Attachment constitutes the first step in virtually all types of biofilm formation, thus numerous studies have focused on preventing bacterial adherence. Efforts have been made to inhibit assembly of different types of pili, through the use of pilicides, which are compounds rationally designed to interfere with export of the corresponding pilin subunits. Pilicides were shown to inhibit biofilm formation in vitro by $50 \%$, at concentrations as low as $3 \mu \mathrm{M}$ [59]. Similar compounds have been shown to be effective against curli (curlicides), inhibiting in vitro curli biogenesis and biofilm formation [60].

\section{Conclusion}

The majority of microbes are able to develop multicellular biofilm communities. These communities are composed of subpopulations of different cell types that provide additional benefits to the cells inside the biofilm. Formation of biofilm enables bacterial pathogens to colonize a wide variety of host niches and persist in harsh environments, making their eradication particularly difficult. Prolonged use of antimicrobial agents enables these pathogens to be more resistant to antibiotics. Novel strategies, designed for dispersal and inhibition of biofilm are reported. The role of biofilms in disease is becoming understandable. However, the need for more effective biofilm dissolution treatments is still fundamental.

\section{References}

1. Sutherland IW (2005) Polysaccharides from microorganisms, plants and animals. Biopolymers Online.

2. Flemming H, Wingender J (2010) The biofilm matrix. Nat Rev Microbiol 8: 623-33.

3. Maria K, Maria H, Scott J (2014) Bacterial Biofilms: Development, Dispersal, and Therapeutic Strategies in the Dawn of the Postantibiotic Era. Cold Spring Harbor Laboratory Press.

4. Tomas B, Oana C, Molin S, Michael G, Niels H (2013) Applying insights from biofilm biology to drug development -can a new approach be developed? Nature Reviews Drug Delivery 12: 791-808.

5. Paraje M (2011) Antimicrobial resistance in biofilms. Science against microbial pathogens: communicating current research and technological advances.

6. Vlamakis H, Aguilar C, Losick R, Kolter R (2008) Control of cell fate by the formation of an architecturally complex bacterial community. Genes Dev 22: $945-53$.

7. Hall-Stoodley L, Costerton J, Stoodley P (2004) Bacterial biofilms: from the natural environment to infectious diseases. Nat Rev Microbiol 2: 95-108.

8. Marlow V, Porter M, Hobley L, Kiley T, Jason R, et al. (2014) Phosphorylated DegU Manipulates Cell Fate Differentiation in the Bacillus subtilis Biofilm. J Bacteriol 196: 16-27.

9. Prakash B, Veeregowda B, Krishnappa G (2003) Biofilms: A survival strategy of bacteria. Current Sci, 85: $1299-307$.

10. Daniel L, Vlamakis H, Kolter R (2010) Biofilms. Cold Spring Harb Perspect Biol 2: a000398.

11. Hjortsø, Martin A, Joseph W (1995) Cell Adhesion: Fundamentals and Biotechnological Applications. New York, USA.

12. Lennox J (2011) Biofilm Development. Biofilms: The Hypertextbook.

13. Marshall KC (1992) Biofilms: an overview of bacterial adhesion, activity, and control at surfaces. ASM News 58: $202-7$.

14. Costerton J, Stewart P, Greenberg E (1999) Bacterial biofilms: A common cause of persistent infections. Science 284: 1318-22.

15. Mittelman M (1996) Biological fouling of purified-water systems: Part 3, Treatment of Micro-contamination 4: 30-40.

16. Patel R (2005) Biofilms and antimicrobial resistance. Clin Orthop Relat Res 437: 41-7.

17. Costerton W, Veeh R, Shirtliff M, Pasmore M, Post C, et al. (2003) The application of biofilm science to the study and control of chronic bacterial infections. J Clin Invest 112:1466-77.

18. Donlan RM, Costerton JW (2002) Biofilms: survival mechanisms of clinically relevant microorganisms. Clin Microbiol Rev 15: 167-93. 
19. Lewis K (2005) Persister cells and the riddle of biofilm survival. Biochemistry 70: 267-74.

20. Keren I, Kaldalu N, Spoering A, Wang YP, Lewis K (2004) Persister cells and tolerance to antimicrobials. FEMS Microbiol Lett 230 : $13-8$.

21. Lewis K (2001) Riddle of biofilm resistance. Antimicrob Agents Chemother 45: 999-1007.

22. Steven LP, Bowler GP (2004) Biofilms and their potential role in wound healing 16.

23. Shigeta M, Tanaka G, Komatsuzawa H, Sugai M, Suginaka H, et al. (1997) Permeation of antimicrobial agents through Pseudomonas aeruginosa biofilms: A simple method. Chemotherapy (Tokyo) 43: 340-5.

24. Hengge-Aronis R (1996) Regulation of gene expression during entry into stationary phase. In: Neidhart FC, et al. (eds). Escherichia coli and Salmonella: Cellular and Molecular Biology. Washington DC: ASM Press 1497-512.

25. Brown MR, Barker J (1999) Unexplored reservoirs of pathogenic bacteria: Protozoa and biofilms. Trends Microbiol 7: 46-50.

26. Gilbert P, Das J, Foley I (1997) Biofilms susceptibility to antimicrobials. Adv Dent Res 11: 160-7.

27. Rehm B (2010) Bacterial polymers: Biosynthesis, modifications and applications. Nat Rev Microbiol 8: 578-92.

28. Nwodo U, Ezeikel G, Okoh A (2012) Bacterial Exopolysaccharides: Functionality and Prospects. Int J Mol Sci 13: $14002-15$.

29. Kokare C, Chakraborty S, Khopade A, Mahadik K (2009) Biofilm: Importance and Applications. Indian J Biotech 8: $159-68$.

30. Høiby N, Bjarnsholt T, Givskov M, Molin S, Ciofu O (2010) Antibiotic resistance of bacterial biofilms. Intr J Antimicr Agents 35: $322-32$.

31. Kolenbrander P, Palmer J (2004) Human oral bacterial biofilms. In: Ghannoum MA, O’Toole GA, eds. Microbial biofilms. Washington, DC: ASM Press.

32. Høiby N, Döring G, Schiøtz P (1986) The role of immune complexes in the pathogenesis of bacterial infections. Annu Rev Microbiol 40: $29-53$.

33. Werner E, Roe F, Bugnicourt A, Franklin M, Heydorn A, et al. (2004) Stratified growth in Pseudomonas aeruginosa biofilms. Appl Environ Microbiol 70: $6188-96$.

34. Skandamis P, Stopforth J, Ashton L, Geornaras I, Kendall P, et al. (2009) Escherichia coli O157:H7 survival, biofilm formation and acid tolerance under simulated slaughter plant moist and dry conditions. Food Microbiol 26: 112-9.

35. Otto M (2008) Staphylococcal biofilms. Curr Top Microbiol Immunol 322: 207-28.

36. Tyler D, Cortney E, John M, Tammy K (2014) Hiding in plain sight: interplay between staphylococcal biofilms and host immunity. Front Immunol 5: 37.

37. ten Cate JM (2006) Biofilms, a new approach to the microbiology of dental plaque. Odontology 94: 1-9.

38. Marsh P, Devine D (2011) How is the development of dental biofilms influenced by the host? J Clin Periodontol 38: 28-35.

39. Lamont R, Jenkinson H (1998) Life below gum line: pathogenic mechanisms in Porphromonas gingivalis. Microbiol Mol Rev 62: $1244-63$.

40. Oggioni MR, Trappetti C, Kadioglu A, Cassone M, Iannelli F, et al. (2006) Switch from planktonic to sessile life: a major event in pneumococcal pathogenesis. Mol. Microbiol 61: 1196-210.

41. Michod RE, Bernstein H, Nedelcu AM (2008) Adaptive value of sex in microbial pathogens. Infect Genet Evol 8: 267-85.

42. Wei H, Håvarstein L (2012) Fratricide is essential for efficient gene transfer between pneumococci in biofilms. Appl Environ Microbiol 78: $5897-905$.

43. Rumbaugh K, Griswold J, Hamood A (2000) The role of quorum sensing in the in vivo virulence of Pseudomonas aeruginosa. Microbes Infect 2: 1721-31.

44. Rumbaugh K, Griswold J, Iglewski B, Hamood A (1999) Contribution of quorum sensing to the virulence of Pseudomonas aeruginosa in burn wound infections. Infect Immun 67: 5854-62.

45. Davies D, Parsek J, Pearson JP, Iglewski B, Costerton J, et al. (1998) The involvement of cell-to-cell signals in the development of a bacterial biofilm. Science 280: 295-8.

46. Murga R, Forster S, Brown E, Pruckler J, Fields B, et al. (2001) Role of biofilms in the survival of Legionella pneumophila in a model potable-water system. Microbiology 147: 3121-6.

47. Swanson M, Hammer B (2000) Legionella pneumophila pathogesesis: a fateful journey from amoebae to macrophages. Annual Rev Microbiol 54: 567-613.

48. Dror N, Mandel M, Hazan Z, Lavie G (2009) Advances in Microbial Biofilm Prevention on Indwelling Medical Devices with Emphasis on Usage of Acoustic Energy 9: 2538-54.

49. Vasilev K, Cook J, Griesser H (2009) Antibacterial surfaces for biomedical devices. Expert Rev Med Devices 6: 553-67.

50. Jansen B, Kohnen W (1995) Prevention of biofilm formation by polymer modification. J Ind Microbiol 15: 391-6.

51. Meiron T, Saguy I (2007) Adhesion Modeling on Rough Low Linear Density Polyethylene. J Food Sci 72: E485-91.

52. Jass J, Surman S, James T (2009) Medical biofilms: detection, prevention, and control. Top academic publications 2: 305.

53. Qin Z, Yang L, Qu D, Molin S, Tolker-Nielsen T (2009) Pseudomonas aeruginosa extracellular products inhibit staphylococcal growth, and disrupt established biofilms produced by Staphylococcus epidermidis. Microbiology 155: 2148-56.

54. Rendueles O, Kaplan J, Ghigo J (2013) Antibiofilm polysaccharides. Environ Microbiol 15: 334-46.

55. Guiton PS, Hung CS, Kline KA, Roth R, Kau AL, et al. (2009) Contribution of autolysin and Sortase A during Enterococcus faecalis DNA-dependent biofilm development. Infect Immun 77: 3626-38.

56. Kaplan JB (2010) Biofilm dispersal: Mechanisms, clinical implications, and potential therapeutic uses. J Dent Res 89: 205-18.

57. Shanks R, Sargent J, Martinez R, Graber M, O’Toole G (2006) Catheter lock solutions influence staphylococcal biofilm formation on abiotic surfaces. Nephrol Dial Transpl 21: 2247-55.

58. Kharidia R, Liang J (2011) The activity of a small lytic peptide PTP-7 on Staphylococcus aureus biofilms. J Microbiol 49: 663-8.

59. Berg V, Das P, Chorell E, Hedenstrom M, Pinkner JS, et al. (2008) Carboxylic acid isosteres improve the activity of ring-fused 2-pyridones that inhibit pilus biogenesis in E. coli. Bioorg Med Chem Lett 18: 3536-40.

60. Cegelski L, Pinkner J, Hammer N, Cusumano C, Hung C, et al. (2009) Small-molecule inhibitors target Escherichia coli amyloid biogenesis and biofilm formation. Nat Chem Biol 5: 913-9. 